\title{
Microwave Sensor Network for Quantitative Characterization of Targets: A Proof-of-Concept
}

\author{
Alessandro Fedeli \\ Department of Electrical, Electronic, \\ Telecommunications Engineering, and Naval Architecture \\ University of Genoa \\ Genoa, Italy \\ alessandro.fedeli@unige.it
}

Matteo Pastorino

Department of Electrical, Electronic,

Telecommunications Engineering, and Naval Architecture

University of Genoa

Genoa, Italy

matteo.pastorino@unige.it

\author{
Gian Luigi Gragnani \\ Department of Electrical, Electronic, \\ Telecommunications Engineering, and Naval Architecture \\ University of Genoa \\ Genoa, Italy \\ gianluigi.gragnani@unige.it \\ Andrea Randazzo \\ Department of Electrical, Electronic, \\ Telecommunications Engineering, and Naval Architecture \\ University of Genoa \\ Genoa, Italy \\ andrea.randazzo@unige.it
}

\begin{abstract}
The possible use of a microwave sensor network for quantitatively reconstructing the electromagnetic properties of unknown targets is considered in this paper. In particular, a set of microwave sensors is used to measure the z-component of the electric field in a free-space scenario. The resulting field measurements are then processed by means of an inversescattering based technique, which provides an estimated map of the dielectric properties of the targets eventually present in the area under investigation. Preliminary numerical results in a simulated environment are shown in order to initially assess the feasibility of the proposed approach.
\end{abstract}

Keywords-Sensor networks, microwaves, inverse scattering.

\section{INTRODUCTION}

Wireless sensor networks (WSNs) are nowadays exploited in many different environments and applicative fields [1]-[4]. The recent developments in the internet-of-things (IoT) paradigm have further pushed the research activities in this area [5], [6]. A possible use of wireless sensor networks, with strong applicative fallbacks, is the detection and localization of targets and people [7], [8]. However, such a localization is usually performed in a qualitative way. In fact, starting from the received signal strength indication (RSSI) that can be measured by several wireless devices (such as access points or mobile phones), an estimation of the target location is provided, usually without information that can be useful to discriminate the target type (e.g., human or object) [9]-[11]. This goal can be pursued by inverse-scattering techniques, which are potentially able to reconstruct the distribution of the dielectric properties (dielectric permittivity and electric conductivity) in a given domain starting from measurements of the electromagnetic field collected by a proper set of wireless sensors [12]-[22].

In this paper, the application of an inverse-scattering technique for obtaining a quantitative characterization of the targets in a known environment is concerned. The inversion method is based on a Newton-conjugate-gradient approach developed in $L^{p}$ Banach spaces, which has been recently proposed in the framework of biomedical imaging [23]. The use of such non-conventional spaces has been found to be able to provide quite good reconstructions in several applications, with less oversmoothing and ringing effects compared to the more standard inversion techniques developed in Hilbert spaces [24], [25].

The paper is organized as follows. In Section II the considered problem is described, outlining a possible strategy for its solution. Initial numerical results in a simplified 2D simulated environment are presented in Section III. Conclusions follow.

\section{Problem Description AND Solution StRategy}

In the considered problem, $N$ microwave transceiver sensors are placed at positions $\mathbf{r}_{n}, n=1, \ldots, N$ in a known scenario, as schematized in Fig. 1. Each of these sensors is able to acquire measurements of the z-component of the electric field, and to share the acquired data with a proper control device, which also takes care of measurement synchronization. Moreover, each sensor has the possibility of radiating a time-harmonic electromagnetic field characterized by a fixed frequency $f$. A single device operates in transmission mode at a time, while all the remaining $N-1$ ones are exploited to measure the electric field. The acquisition phase is iterated until all the sensors have been utilized in both transmitting and receiving mode.

The goal of the present methodology is to reconstruct, starting from a set of electric field measurements of this kind, the dielectric properties of a predefined region under inspection, denoted as the investigation area $\mathcal{J}$ (which lies on the $x y$ plane). Only non-magnetic materials are considered in this paper (i.e., with their magnetic permeability $\mu$ equal to the vacuum value, $\left.\mu_{0} \simeq 1.26 \times 10^{-6} \mathrm{H} / \mathrm{m}\right)$. A tomographic approach characterized by TM-z electromagnetic fields and object properties invariant along the $\mathrm{z}$ axis is presented here for the sake of simplicity, but its three-dimensional extension can be formulated quite straightforwardly (although the implementation can be non-trivial). 


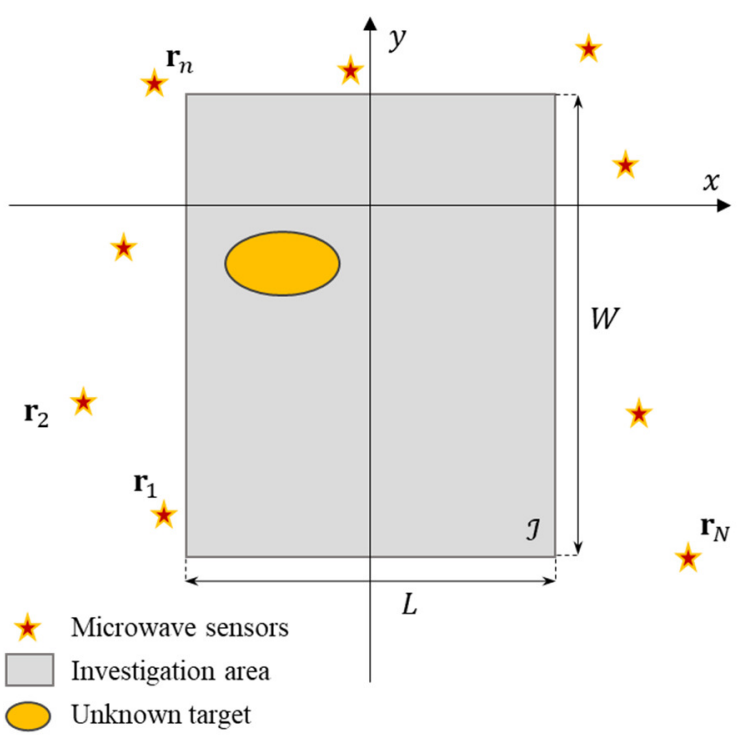

Fig. 1. Quantitative characterization of targets with microwave sensors: schematic configuration of the problem.

As it is well known, the electric field is perturbed by the presence of unknown targets in $\mathcal{J}$, and therefore the measurement of this perturbation with respect to the reference object-free scenario (denoted as the scattered electric field, $E_{s c}$ ) can be exploited to retrieve the properties of the unknown targets [26]. In particular, we are interested in reconstructing the distribution of the complex relative dielectric permittivity, defined as

$$
\epsilon_{r}(\mathbf{r})=\epsilon_{r}^{\prime}(\mathbf{r})-j \frac{\sigma(\mathbf{r})}{2 \pi f \epsilon_{0}}, \quad \mathbf{r} \in \mathcal{J},
$$

where $\epsilon_{r}^{\prime}$ is the real part of the relative dielectric permittivity, $\sigma$ is the effective electric conductivity, and $\epsilon_{0} \simeq 8.85 \times$ $10^{-12} \mathrm{~F} / \mathrm{m}$ indicates the dielectric permittivity of the vacuum. It is worth noting that, in the practical implementation of the approach, the investigation area $\mathcal{J}$ is partitioned into $I$ square cells $\mathcal{J}_{i}, i=1, \ldots, I$, centered at $\mathbf{r}_{i}^{\mathcal{J}}$, in which both fields and dielectric properties can be assumed constant (i.e., piecewise constant basis functions are used for discretizing the continuous model).

For each position of the transmitting device, the $Z$ component of the scattered field $E_{S c}$ is assumed to be known at the locations of the other sensors working in receiving mode. For instance, assuming that the first sensor (located at $\mathbf{r}_{1}$ ) is the only one in transmitting mode, the relationship between the $E_{S c}$ and the dielectric properties inside $\mathcal{J}$ (in discrete settings) is [24]

$$
E_{s c}\left(\mathbf{r}_{n}\right)=\sum_{i=1}^{I} c\left(\mathbf{r}_{i}^{\mathcal{J}}\right) E\left(\mathbf{r}_{i}^{\mathcal{J}}\right) h_{i}\left(\mathbf{r}_{n}\right), \quad n=2, \ldots, N
$$

where $c\left(\mathbf{r}_{i}\right)=\epsilon_{r}\left(\mathbf{r}_{i}\right)-1$ is the contrast function in a freespace background (modeled as vacuum), $E\left(\mathbf{r}_{i}^{\mathcal{J}}\right)$ is the total electric field inside the $i$-th subdomain, and $h_{i}\left(\mathbf{r}_{n}\right)$ is given by [26]

$$
h_{i}\left(\mathbf{r}_{n}\right)=-k_{0}^{2} \int_{\mathcal{J}_{i}} g\left(\mathbf{r}_{n}, \mathbf{r}\right) d \mathbf{r}
$$

where $k_{0}=2 \pi f\left(\mu_{0} \epsilon_{0}\right)^{0.5}$ is the vacuum wavenumber and $g$ is the Green's function of the considered scenario. Defining the vectors $\mathbf{c}=\left[c\left(\mathbf{r}_{1}^{\mathcal{J}}\right), \ldots, c\left(\mathbf{r}_{I}^{\mathcal{J}}\right)\right]^{T}, \mathbf{e}=\left[E\left(\mathbf{r}_{1}^{\mathcal{J}}\right), \ldots, E\left(\mathbf{r}_{I}^{\mathcal{J}}\right)\right]^{T}$, and $\mathbf{e}_{s c}=\left[E_{s c}\left(\mathbf{r}_{2}\right), \ldots, E_{s c}\left(\mathbf{r}_{N}\right)\right]^{T},(2)$ can be expressed as

$$
\mathbf{e}_{s c}=\mathbf{H}_{d t} \operatorname{diag}(\mathbf{c}) \mathbf{e}
$$

where $\mathbf{H}_{d t}$ is a $(N-1) \times I$ matrix whose elements are given by $\left[\mathbf{H}_{d t}\right]_{n, i}=h_{i}\left(\mathbf{r}_{n}\right)$. The vector $\mathbf{e}$ can be retrieved from another equation, which can be found in a similar manner, and is given by

$$
\mathbf{e}=\mathbf{e}_{\text {in }}+\mathbf{H}_{s t} \operatorname{diag}(\mathbf{c}) \mathbf{e}
$$

where $\mathbf{e}_{\text {in }}=\left[E_{\text {in }}\left(\mathbf{r}_{1}^{\jmath}\right), \ldots, E_{\text {in }}\left(\mathbf{r}_{I}^{\jmath}\right)\right]^{T}$ is a vector containing the values of the electric field in the investigation area without the unknown targets, and $\left[\mathbf{H}_{s t}\right]_{r, s}=h_{r}\left(\mathbf{r}_{s}^{J}\right)$, with $r, s=1, \ldots, I$ are the elements of a square matrix of dimension $I$. In the present approach, (4) and (5) are combined together, yielding

$$
\mathbf{e}_{s c}=\mathbf{G}(\mathbf{c})=\mathbf{H}_{d t} \operatorname{diag}(\mathbf{c})\left[\mathbf{I}-\mathbf{H}_{s t} \operatorname{diag}(\mathbf{c})\right]^{-1} \mathbf{e}_{i n}
$$

which is a nonlinear equation with respect to the unknown $\mathbf{c}$, i.e., the vector representing point-by-point the dielectric properties of the investigation area $\mathcal{J}$. Actually, this equation also turns out to be strongly ill-posed, due to the properties of the underlying inverse scattering problem. Consequently, a special care must be used in the development of the inversion procedures, which must be able to address both the nonlinearity and the ill-posedness of the problem.

In this work, the unknown vector $\mathbf{c}$ is found by applying a solution strategy based on a Newton-conjugate-gradient (NCG) iterative technique defined in the mathematical context of $L^{p}$ Banach spaces. The main steps of this algorithm, initially proposed by Bisio et al. in [23], are summarized in Fig. 2. In particular, the adopted solution scheme is formed by a two-loop structure. The external iterations (denoted by the index $l$ ), which represent a standard inexact-Newton scheme, are used to linearize (6) around the currently reconstructed values of the contrast function $\mathbf{c}_{l}$. The solution of the linearized equation is carried out by a non-conventional conjugate-gradient procedure operating in $L^{p}$ Banach spaces, where $p$ is a parameter that can be tuned inside the inversion process. This method acts as an internal loop. Both kind of

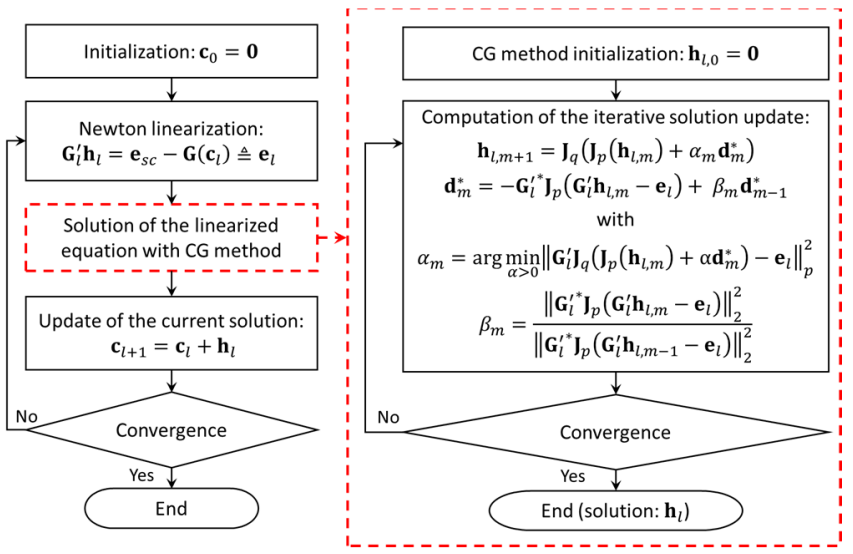

Fig. 2. Flow-chart representation of the main steps of the algorithm employed for the quantitative characterization of dielectric targets. 
iterations are stopped when convergence is reached, i.e., the relative variation of the minimized cost function is under a predefined threshold. More details about the solving procedure can be found in [23].

\section{PReliminary ReSUlts in A Simulated ENVIRONMENT}

A simulated test case has been considered as a proof-ofconcept of the proposed method. A network of $N=15$ microwave sensors is placed in a free-space environment. These sensors are equipped with transceivers operating at the frequency $f=300 \mathrm{MHz}$. As mentioned in the previous Section, each of these sensors in turn act as a transmitter, whereas the $N-1$ others are used to measure the resulting scattered electric field. In this case, the electric field data have been numerically computed by a custom method-of-moment numerical simulator [27], and then a white Gaussian noise with mean value equal to zero and signal-to-noise ratio $S N R=20 \mathrm{~dB}$ has been added in order to simulate more realistic operating conditions.

The considered investigation area $\mathcal{J}$ is a rectangular region whose $x$ and $y$ dimensions are $L=1.6 \mathrm{~m}$ and $W=2.4 \mathrm{~m}$, respectively. However, it is worth noting that the developed approach can be used with arbitrarily shaped domains, too. The forward problem has been solved by discretizing $\mathcal{J}$ with a grid of $I_{f}=48 \times 72$ subdomains, in which each cell is a square with side length $d_{f}=0.033 \mathrm{~m}$. A cylindrical object with rectangular cross section of side lengths $s_{x}=0.5 \mathrm{~m}$ and $s_{y}=0.3 \mathrm{~m}$, characterized by relative dielectric permittivity $\epsilon_{r}^{\prime}=3$ and electric conductivity $\sigma=0.01 \mathrm{~S} / \mathrm{m}$ (i.e., the dielectric properties of dry wood) is centered at the point with coordinates $\mathbf{r}_{c}=(-0.2,-0.8) \mathrm{m}$. The Newton-conjugategradient inversion method has been utilized with the following parameters: fixed $L^{p}$ space exponent $p=1.4$, maximum number of internal and external iterations $M=50$, minimum relative variation of the residual cost function in both loops $\Delta R=0.15$. In the solution of the inverse problem, $\mathcal{J}$ has been partitioned into $I=32 \times 48$ square subdomains with side length equal to $d_{i}=0.05 \mathrm{~m}$.

In the first case study, the microwave sensors (whose positions on the $x y$ plane are detailed in Table I) are uniformly distributed with a spacing of $0.5 \mathrm{~m}$ to each other and aligned with three sides of the investigation area. In other words, they are located on three lines external to the border of $\mathcal{J}$ characterized with $y=0.5 \mathrm{~m}, x=-1 \mathrm{~m}$, and $x=1 \mathrm{~m}$, respectively. The reconstructed distributions of the relative dielectric permittivity and of the electric conductivity obtained with this measurement configuration are shown in Fig. 3, where a red line highlights the actual profile of the target. The cylindrical object appears to be correctly located and characterized, although an overestimation of the value of the dielectric properties can be observed near the center of the target. Some artifacts with low values of permittivity and conductivity appear in the background region, especially close to the target, and are mainly due to the relatively low number of adopted sensors.

The second analyzed case concerns a network of nonaligned sensors, located around the investigation area with variable distances. Their $x y$-plane locations, which have been obtained by perturbing the positions used in the first case study with a displacement up to $20 \mathrm{~cm}$, are reported in Table II. The reconstructed values of the dielectric properties are presented in Fig. 4. From this figure, it is possible to notice that the reconstructed image is very similar to the previous case, where a set of aligned microwave probes has been adopted.

In both cases, the quality of the dielectric characterization has been assessed by means of the relative errors computed in the background area, the target area, and the whole investigation region $\mathcal{J}$. These performance metrics, denoted as $\Gamma_{b}, \Gamma_{t}$, and $\Gamma_{\mathcal{\jmath}}$ respectively, are defined as follows:

$$
\left\{\Gamma_{b}, \Gamma_{t}, \Gamma_{\mathcal{J}}\right\}=\frac{1}{\left\{I_{b}, I_{t}, I\right\}} \sum_{\mathbf{r}_{i} \in\left\{\mathcal{J}_{b}, J_{t}, \mathfrak{J}\right\}}\left|\frac{\epsilon_{r}\left(\mathbf{r}_{i}\right)-\widehat{\epsilon_{r}}\left(\mathbf{r}_{i}\right)}{\widehat{\epsilon_{r}}\left(\mathbf{r}_{i}\right)}\right|
$$

where $\epsilon_{r}\left(\mathbf{r}_{i}\right)$ is the reconstructed value of the relative dielectric permittivity at the point $\mathbf{r}_{i}, \widehat{\epsilon_{r}}\left(\mathbf{r}_{i}\right)$ being the corresponding actual value. Furthermore, $\mathcal{J}_{b}, \mathcal{J}_{t}$ represent the background and the target domains, which contain $I_{b}, I_{t}$ cells, respectively. These errors are reported in Table III for the two considered test cases, i.e., with aligned and non-aligned sensors. As can be seen, the errors are comparable. However, the result with aligned sensors present a slightly lower error on the reconstruction of the target, whereas the reconstruction with non-aligned sensors presents small improvements in the background errors, as well as in the relative error computed on the whole investigation area.

TABLE I. POSITIONS OF THE MicROWAVE SENSORS IN THE FIRST SIMULATED SCENARIO

\begin{tabular}{|c|c|c|c|c|c|c|c|c|}
\hline ID & $\mathbf{x}(\mathbf{m})$ & $\mathbf{y}(\mathbf{m})$ & ID & $\mathbf{x}(\mathbf{m})$ & $\mathbf{y}(\mathbf{m})$ & ID & $\mathbf{x}(\mathbf{m})$ & $\mathbf{y}(\mathbf{m})$ \\
\hline$\# 1$ & -1.00 & 0.50 & $\# 6$ & -1.00 & -2.00 & $\# 11$ & 1.00 & -2.00 \\
\hline$\# 2$ & -0.50 & 0.50 & $\# 7$ & -1.00 & -1.50 & $\# 12$ & 1.00 & -1.50 \\
\hline$\# 3$ & 0 & 0.50 & $\# 8$ & -1.00 & -1.00 & $\# 13$ & 1.00 & -1.00 \\
\hline$\# 4$ & 0.50 & 0.50 & $\# 9$ & -1.00 & -0.50 & $\# 14$ & 1.00 & -0.50 \\
\hline$\# 5$ & 1.00 & 0.50 & $\# 10$ & -1.00 & 0 & $\# 15$ & 1.00 & 0 \\
\hline
\end{tabular}

TABLE II. POSITIONS OF THE MiCROWAVE SENSORS IN THE SECOND Simulated SCENARIO

\begin{tabular}{|c|c|c|c|c|c|c|c|c|}
\hline ID & $\mathbf{x}(\mathbf{m})$ & $\mathbf{y}(\mathbf{m})$ & ID & $\mathbf{x}(\mathbf{m})$ & $\mathbf{y}(\mathbf{m})$ & ID & $\mathbf{x}(\mathbf{m})$ & $\mathbf{y}(\mathbf{m})$ \\
\hline$\# 1$ & -1.05 & 0.40 & $\# 6$ & -0.90 & -2.10 & $\# 11$ & 0.90 & -2.00 \\
\hline$\# 2$ & -0.50 & 0.50 & $\# 7$ & -1.00 & -1.40 & $\# 12$ & 1.00 & -1.60 \\
\hline$\# 3$ & 0.10 & 0.45 & $\# 8$ & -0.95 & -1.10 & $\# 13$ & 1.10 & -1.20 \\
\hline$\# 4$ & 0.50 & 0.55 & $\# 9$ & -0.85 & -0.50 & $\# 14$ & 0.85 & -0.60 \\
\hline$\# 5$ & 1.10 & 0.45 & $\# 10$ & -1.00 & 0.10 & $\# 15$ & 0.95 & 0.20 \\
\hline
\end{tabular}

TABLE III. RELATIVE ERRORS ON THE DIELECTRIC CHARACTERIZATION

\begin{tabular}{|c|c|c|c|}
\hline \multirow{2}{*}{ Case } & \multicolumn{3}{|c|}{ Relative Characterization Errors } \\
\cline { 2 - 4 } & $\begin{array}{c}\text { Background area, } \\
\boldsymbol{\Gamma}_{\boldsymbol{b}}\end{array}$ & Target area, $\boldsymbol{\Gamma}_{\boldsymbol{t}}$ & $\begin{array}{c}\text { Whole inspection } \\
\text { domain, } \boldsymbol{\Gamma}_{\boldsymbol{J}}\end{array}$ \\
\hline$\# 1$ & 0.193 & 0.402 & 0.201 \\
\hline$\# 2$ & 0.160 & 0.429 & 0.170 \\
\hline
\end{tabular}




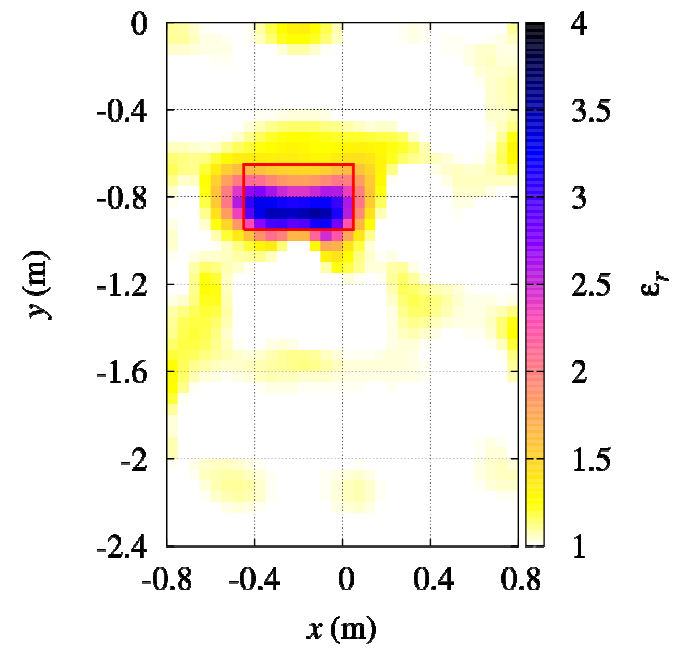

(a)

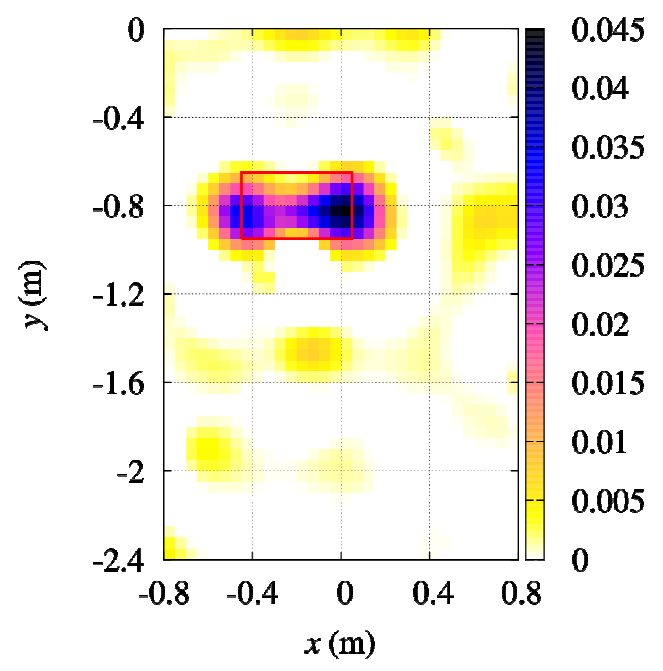

(b)

Fig. 3. Reconstructed distributions of the (a) relative dielectric permittivity, and (b) electric conductivity of the investigation region, obtained in the first simulated scenario (case \#1, aligned microwave sensors).

\section{CONCLUSIONS}

Wireless sensor networks are nowadays considered for a large number of both experimented and potential applications. One of these potential applications is the quantitative characterization of dielectric targets in known environments. In this paper, such a problem is addressed considering a network of microwave transceivers located around a rectangular investigation area. The acquired scattered-field measurements are used as input data for a Newton-conjugategradient algorithm that provides a full dielectric characterization of the scenario. Preliminary numerical results in a simplified scenario have been presented to assess the proposed methodology. Future developments will include the assessment of the approach in more realistic conditions, e.g., in presence of errors in the positions of the sensor-network elements and using different frequency bands (e.g., the WiFi one), and the extension to phaseless inverse-scattering techniques, which are simpler to be applied in real-world situations since the phase reference is not required during measurements. The experimental validation of the proposed technique will be considered, too.

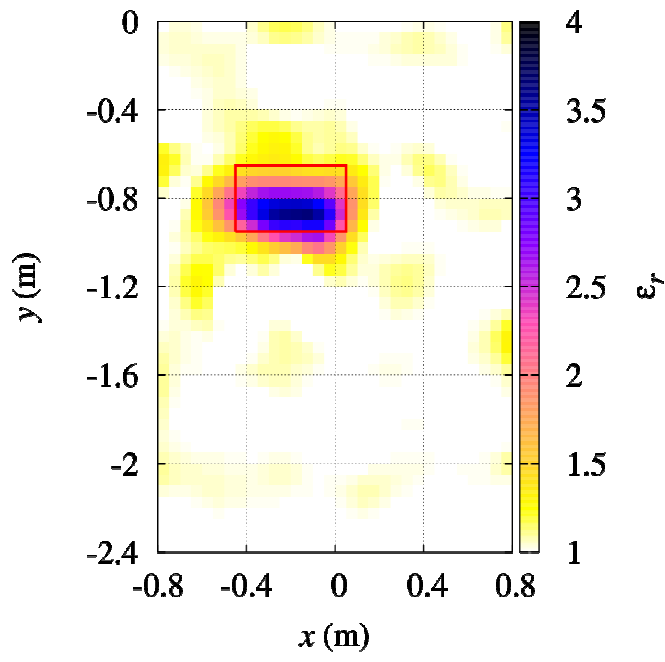

(a)

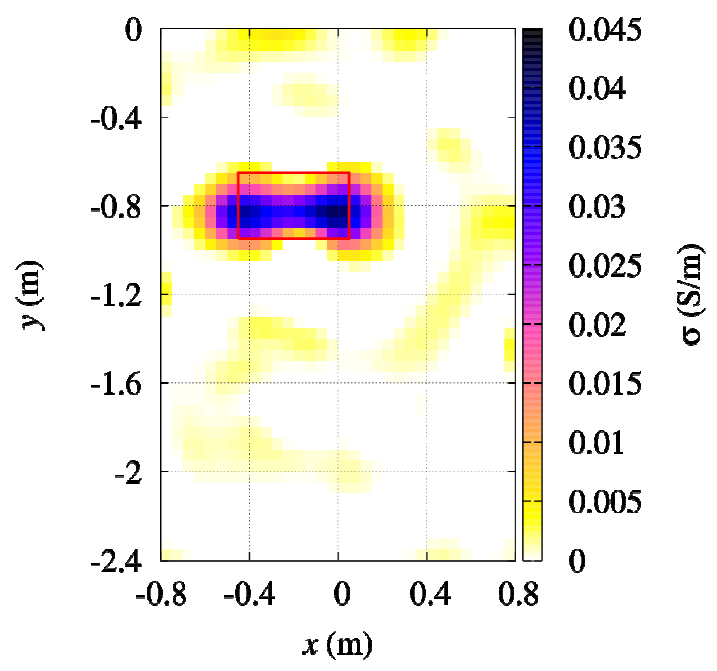

(b)

Fig. 4. Reconstructed distributions of the (a) relative dielectric permittivity, and (b) electric conductivity of the investigation region obtained in the second simulated scenario (case \#2, non-aligned microwave sensors).

\section{REFERENCES}

[1] E. D. Skiani, S. A. Mitilineos, and S. C. A. Thomopoulos, "A study of the performance of wireless sensor networks operating with smart antennas," IEEE Antennas Propag. Mag., vol. 54, no. 3, pp. 50-67, Jun. 2012.

[2] X. Tan, Z. Sun, and I. F. Akyildiz, "Wireless underground sensor networks: MI-based communication systems for underground applications.," IEEE Antennas Propag. Mag., vol. 57, no. 4, pp. 74-87, Aug. 2015.

[3] S. Ali et al., "SimpliMote: A wireless sensor network monitoring platform for oil and gas pipelines," IEEE Syst. J., vol. 12, no. 1, pp. 778-789, Mar. 2018.

[4] Q. Zhang, J. W. Wan, D. D. Wang, J. Y. Chen, and D. H. Wang, "Sparsity-incorporated secure localisation for wireless sensor networks," Electron. Lett., vol. 53, no. 9, pp. 629-631, 2017.

[5] S. M. A. Oteafy and H. S. Hassanein, "Big sensed data: Evolution, challenges, and a progressive framework," IEEE Commun. Mag., vol. 56, no. 7, pp. 108-114, Jul. 2018.

[6] Z. Sheng, D. Tian, and V. C. M. Leung, "Toward an energy and resource efficient internet of things: A design principle combining computation, communications, and protocols," IEEE Commun. Mag., vol. 56, no. 7, pp. 89-95, Jul. 2018.

[7] J. Luo, Z. Zhang, C. Liu, and H. Luo, "Reliable and cooperative target tracking based on wsn and WiFi in indoor wireless networks," IEEE Access, vol. 6, pp. 24846-24855, 2018. 
[8] X. Fang, L. Nan, Z. Jiang, and L. Chen, "Multi-channel fingerprint localisation algorithm for wireless sensor network in multipath environment," IET Commun., vol. 11, no. 15, pp. 2253-2260, 2017.

[9] P. K. Sahu, E. H. Wu, and J. Sahoo, "DuRT: Dual RSSI trend based localization for wireless sensor networks," IEEE Sens. J., vol. 13, no. 8, pp. 3115-3123, Aug. 2013.

[10] I. Bisio et al., "A trainingless WiFi fingerprint positioning approach over mobile devices," IEEE Antennas Wireless Propag. Lett., vol. 13, pp. 832-835, 2014.

[11] R. Niu, A. Vempaty, and P. K. Varshney, "Received-signal-strengthbased localization in wireless sensor networks," Proc. IEEE, vol. 106, no. 7, pp. 1166-1182, Jul. 2018.

[12] L. Li, L. G. Wang, J. Ding, P. K. Liu, M. Y. Xia, and T. J. Cui, “A probabilistic model for the nonlinear electromagnetic inverse scattering: TM case," IEEE Trans. Antennas Propag., vol. 65, no. 11, pp. 5984-5991, Nov. 2017.

[13] A. Desmal and H. Bağc1, "Sparse nonlinear electromagnetic imaging accelerated with projected steepest descent algorithm," IEEE Trans. Geosci. Remote Sens., vol. 55, no. 7, pp. 3810-3822, Jul. 2017.

[14] F. Boero et al., "Microwave tomography for the inspection of wood materials: imaging system and experimental results," IEEE Trans. Microw. Theory Techn., vol. 66, no. 7, pp. 3497-3510, Jul. 2018.

[15] Y. Zhong, M. Lambert, D. Lesselier, and X. Chen, "A new integral equation method to solve highly nonlinear inverse scattering problems," IEEE Trans. Antennas Propag., vol. 64, no. 5, pp. 17881799, May 2016.

[16] M. Salucci et al., "Three-dimensional electromagnetic imaging of dielectric targets by means of the multiscaling inexact-Newton method," J. Opt. Soc. Am. A, vol. 34, no. 7, p. 1119, Jul. 2017.

[17] I. Bisio et al., "A numerical study concerning brain stroke detection by microwave imaging systems," Multimed. Tools Appl., vol. 77, no. 8, pp. 9341-9363, Apr. 2018.
[18] A. Randazzo, "Swarm optimization methods in microwave imaging," Int. J. Microw. Sci. Technol., vol. 2012, pp. 1-12 (491713), 2012.

[19] A. Abubakar, T. M. Habashy, and G. Pan, "Microwave data inversions using the source-receiver compression scheme," IEEE Trans. Antennas Propag., vol. 60, no. 6, pp. 2853-2864, Jun. 2012.

[20] M. Pastorino, "Short-range microwave inverse scattering techniques for image reconstruction and applications," IEEE Trans. Instrum. Meas., vol. 47, no. 6, pp. 1419-1427, Dec. 1998.

[21] D. S. Shumakov and N. K. Nikolova, "Fast quantitative microwave imaging with scattered-power maps," IEEE Trans. Microw. Theory Techn., vol. 66, no. 1, pp. 439-449, Jan. 2018.

[22] S. Caorsi, A. Massa, M. Pastorino, and A. Randazzo, "Electromagnetic detection of dielectric scatterers using phaseless synthetic and real data and the memetic algorithm," IEEE Trans. Geosci. Remote Sens., vol. 41, no. 12, pp. 2745-2753, Dec. 2003.

[23] I. Bisio et al., "Brain stroke microwave imaging by means of a Newtonconjugate-gradient method in Lp Banach spaces," IEEE Trans. Microw. Theory Techn., vol. 66, no. 8, pp. 3668-3682, Aug. 2018.

[24] C. Estatico, A. Fedeli, M. Pastorino, and A. Randazzo, "Microwave imaging of elliptically shaped dielectric cylinders by means of an Lp Banach-space inversion algorithm," Meas. Sci. Technol., vol. 24, no. 7, p. 074017, Jul. 2013.

[25] C. Estatico, A. Fedeli, M. Pastorino, and A. Randazzo, "A multifrequency inexact-Newton method in Lp Banach spaces for buried objects detection," IEEE Trans. Antennas Propag., vol. 63, no. 9, pp. 4198-4204, Sep. 2015.

[26] M. Pastorino and A. Randazzo, Microwave imaging. Methods and applications. Boston, MA: Artech House, 2018.

[27] R. F. Harrington, Field computation by moment methods. Piscataway, NJ: IEEE, 1993. 